\title{
Designing for change and reusability - Using XML, XSL, and MPEG-7 for developing professional health Information systems
}

\author{
Ad Emmen ${ }^{1}$ \\ 1 Genias Benelux bv, James Stewartstraat 248, NL-1325 JN \\ Almere, The Netherlands, emmendgenias.nl
}

\begin{abstract}
With each new generation of devices, there is a tendency to develop information systems completely from the start, because the old ones were optimised for the previous generation of devices. XML experience has now reached a stage at which it is possible to define guidelines on designing information systems that are device independent, and that have adaptable user interfaces. We show some of these guidelines illustrated by the design of health care provider Internet accessible information systems. We report on a marketplace for medical multimedia objects, called the GridSET Exchange, that is completely based on existing and emerging XML standards. It explores the possibility of using the new MPEG-7 standard for these type of applications. We use a semiautomatic editor generator to construct editors from MPEG-7 fragments.
\end{abstract}

\section{Introduction}

With each new generation of devices, there is a tendency to develop information systems completely from the start, because the old ones were optimised for the previous generation of devices. Hence, there is for instance already a large amount of HTML-coded legacy systems even though the Internet is young. Much effort has been put into systems like WAP/WML that did not live up to their expectations.

The original promise of XML way back in 1998 was that everything would become easy, because there was now only one format. It was not quite that simple, of course. But XML experience has now reached a stage at which it is possible to define guidelines on designing information systems that are device independent, and that have adaptable user interfaces.

We show some of these guidelines illustrated by the design of health care provider Internet accessible information systems. In our system, all data, not only the information itself, but also the site lay-out, the configuration, the user management, is stored as XML. To create views for a user, be it an edit screen, or a list for display on a channel, XSLT is used. One can say that XSLT is the technology used to convert the $\mathrm{XML}$ information data into knowledge[4].

Because we do no exactly know what will be for instance the parameters of the $3 \mathrm{G}$ device on which our information has to be displayed, we store our intended lay-out as 
XSL-FO. XSL-FO turns out to be powerful enough not only to be used as a device independent page description language, but also as a useful format to describe screens.

The first industry standards are now emerging, that were designed after experience with XML was gained, as opposed to the first generation of industry standards that merely consisted of translating some previous existing standards into XML.

In our system we built in parts based on the new MPEG-7 standard. MPEG-7 is an ISO standard for describing meta-data on multi-media objects. MPEG-7 is very elaborate, not only describing the multi-media objects, including for instance video, sound and pictures, themselves, but also has tools for creating collections and describing user information, including browser and search preferences. As such, it provides a possible useful technology for $3 \mathrm{G}$ devices too.

Our system is built on top of G-VMP, the Genias Virtual Media Publisher[1]. GVMP itself is an elaborate content management system focused on publishing magazines, newsletters, for web, print, e-mail and channels, and managing community sites.

We describe the implementation of this system for the GridSET Exchange. This is part of the WebSET project [2]. The WebSET project aims to produce a standardised suite of interactive three-dimensional educational tools, delivered across the WWW. The major focus is put on the use of open technology and standards, as well as the production of learning components which can be used as building blocks for further development in a wide range of application areas, such as surgical training and physiological education. WebSET is supported by the European Commission under contract number IST-1999-10632.

The GridSET Exchange will be used to market reusable learning objects - 3-D models, and course-modules.

People must be able to put these on the web site in a secure way. They should say who can use them under which conditions, and have a way to view the information on the usage of their objects.

On the other hand potential customers will be able to browse the site, get a preview and acquire an object. When payment is necessary, the system will take care of that too.

Although the name is GridSET Exchange, it is in fact a catalogue based market place [3].

\section{GridSET Exchange design}

The GridSET Exchange's core consists of a repository where the reusable learning objects are stored. At a second level, a meta-data catalogue is build. This catalogue contains information on all the objects, on the creators and the conditions of use. At a third level, the GridSET site engine can be used to access the catalogue, for entering new objects in the repository with simultaneous update to the catalogue; for searching, and for acquiring objects.

Note that this design follows the standard three level-approach of knowledge based systems [4]:

The lowest level is the data level, in our case the objects. 
At the second level, by adding meta-data, the data is turned into information, in our case the catalogue.

At the third level, the information is turned into knowledge in our case for instance a search-engine allows for sophisticated queries.

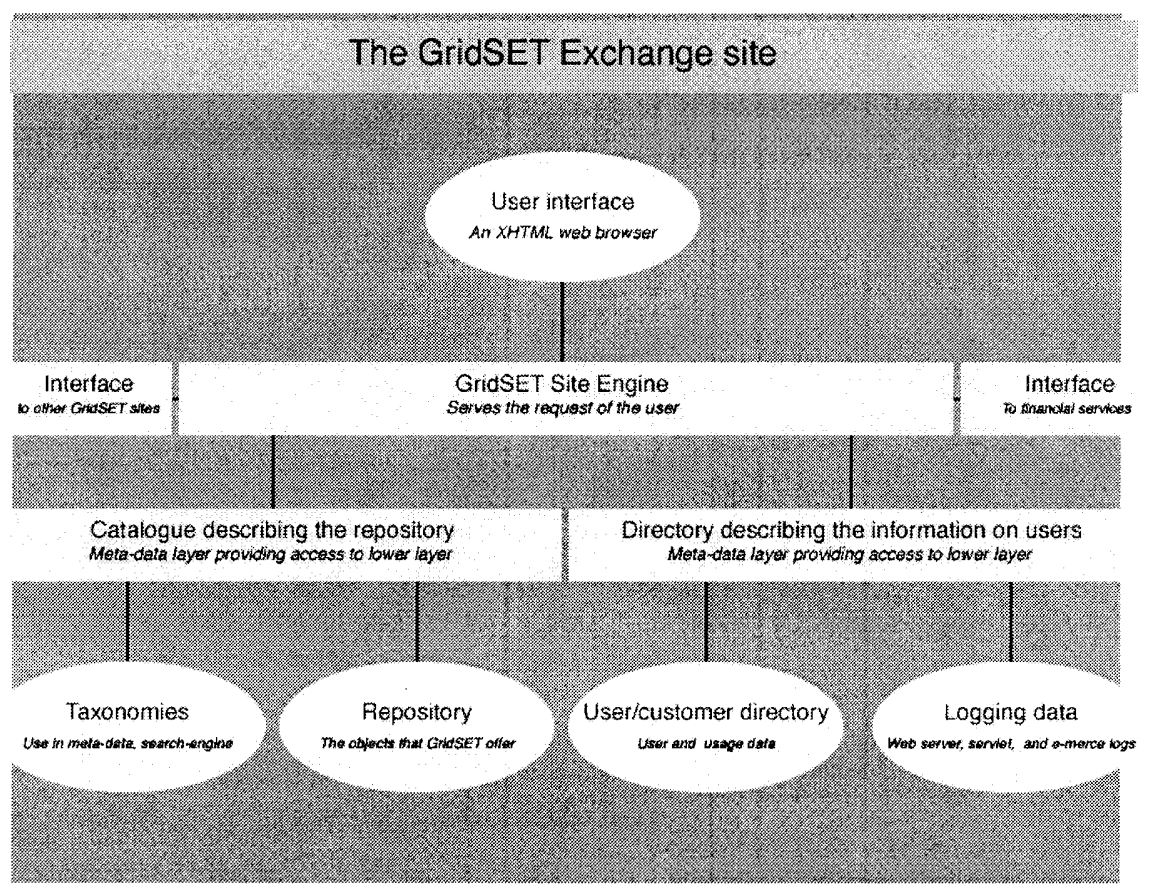

Apart from the repository, the data-level also contains the taxonomies that can be used to augment the information at the meta-data level.

Notice, that, although, strictly spoken, taxonomies are also meta-data, for GridSET, they are data until integrated at the next level. This general also allows taxonomies to have their own format. They can reside on the GridSET server, but can also be maintained elsewhere.

The nexts item at the data level are the directories holding the data on the users, and their usage. This includes data on who they are, access rights, selling and buying data, etc. Again, the separate layer allows to use other types of directory services too.

The last item in the data level consists of logging information. The activities of the server, servlets, and users will be logged.

The information level has two main items. The meta-data catalogue describing the repository is the most important one. It contains all information needed to access the repository. Taxonomies, glossaries and perhaps other information will be integrated to enrich the catalogue. MPEG-7 [5] will be used to implement most, if not all of the catalogue. MPEG-7 features elements to describe the Content of an item in the repository. It contains Collection elements that will used for grouping. Controlled vo- 
cabularies will be used to integrate external taxonomies, and Creation-elements to describe the information on the person/organisation who did create it.

MPEG-7 is a recently defined ISO standard that describes meta-data about multimedia objects. Apart from detailed descriptions of the properties, information on creation, ownership and allowable use can be specified. Also information on financial results, users and user profiles can be described. Outside taxonomies can be easily included. Although designed by the media researchers and companies, it provides definition schemes that can be useful for Grid information systems too. The design of the GridSET Exchange is an exploration in this field.

The second item in the information level, is the directory containing user/usage information. This can be relatively lightweight, because we do not need that much information for each users, apart from the usual information, that can be implemented using MPEG-7, we need to represent access privileges and usage records.

The knowledge layer is powered by the GridSET Engine. It provides the user a view on the information on the GridSET system and tools to manipulate and search it.

In addition GridSET engine can have two external interfaces:

- An interface to financial services.

- An interface to other GridSET sites.

The financial services check creditability of a user and execute payments. GridSET will use one of the existing services [6] that allow exchange of information by means of XML-based records.

The interface to other GridSET servers can be used to send requests to see whether objects not present in the current repository are perhaps retrievable from somewhere else. Also it should handle incoming requests. The communication protocol implementation under investigation are a DNS model, flocking as used in Condor, or a centralised view of catalogues.

The interface to the other GridSET sites, allows for creating a peer-to-peer based network of GridSET Exchanges. A complete description of this interface is outside the scope of this paper.

The 3-layered approach chosen, allows to create a completely distributed GridSET Exchange system. The repository, for instance, does not have to be

on the same computer as the meta-data catalogue. The same holds for the GridSET engine. Also the repository itself can be distributed over many

computers, as can the meta-data catalogue.

In general, communication between the components of the system takes place by exchanging XML-messages. Location or access is performed through the URL naming scheme.

\section{The GridSET Repository}

The GridSET repository contains the reusable learning objects GridSET wants to make available. What can these be? 3-D models, written in VRML or X3D are amongst them. These can be for instance spine models, or torso models. Course modules, or course module fragments are the second type of items. These XML-files can 
be used as parts of (new) training courses. Third category consists of simulator programmes written in Java, that can be combined with $3-\mathrm{D}$ modules and integrated in training courses.

Additional items can be images, for instance a clinician at work, in any of the usual formats, for instance in JPEG, movies, form instance animations of real operations in for instance MPEG-2, and multi-media presentations in for instance SMIL.

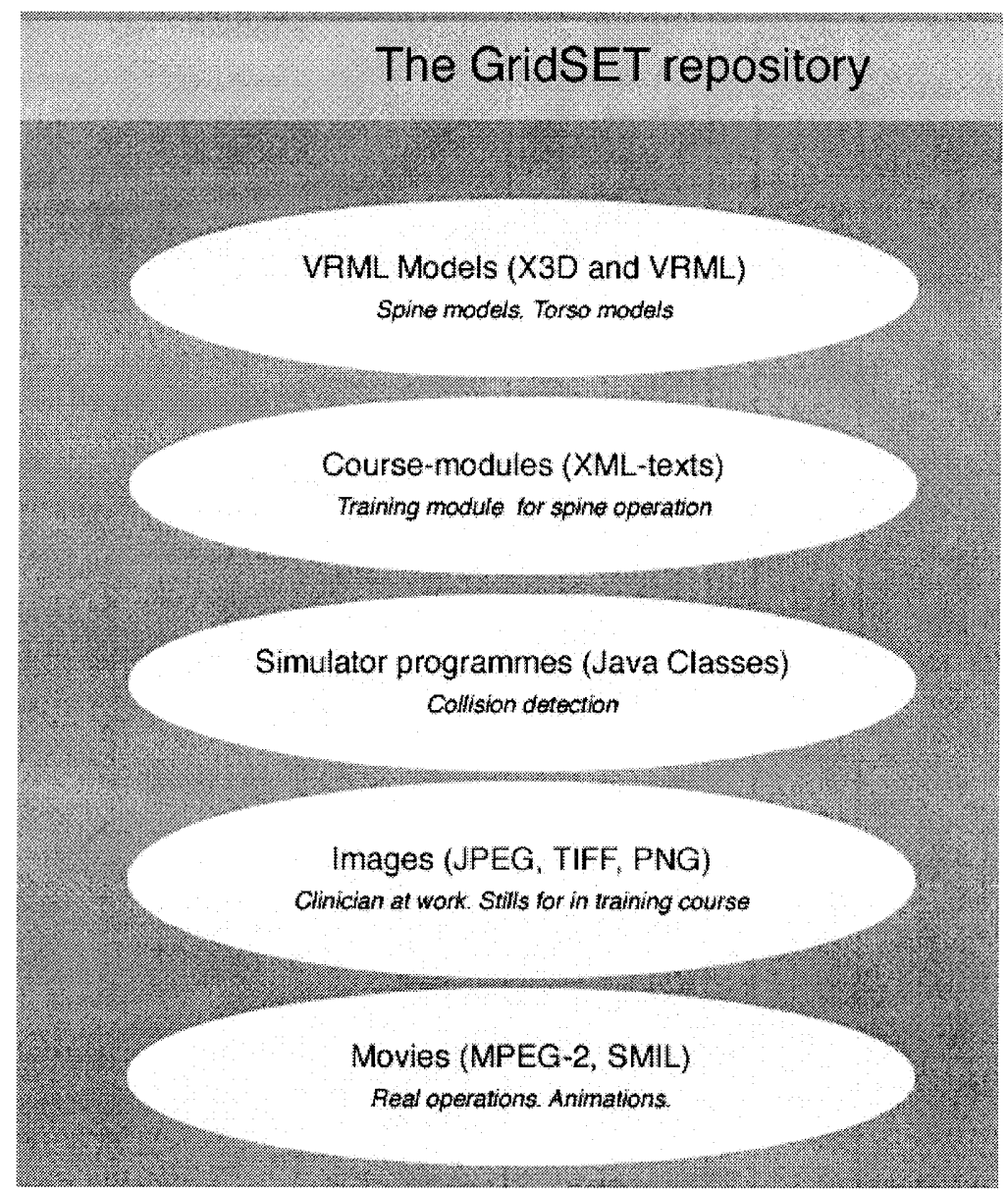

The implementation of the Repository is straight forward. Only a storage mechanism with URL type of access with appropriate compression is needed, while the meta-data layer takes care of all the information attached to the objects. A full blown relational database system, for instance, would add too much overhead providing functionality that is already provided by the meta-data layer. 


\section{The GridSET Site Engine}

The GridSET Site Engine serves requests (mainly http requests) from a web browser. Part of these requests are for the static part of the web site that can be handled by a standard web server, the majority of requests will be dynamic in nature and serviced by a Servlet Engine.

The meta-data layer, consisting of XML-documents only, can be completely handled by XSLT-transformation sheets and formatting stylesheets.

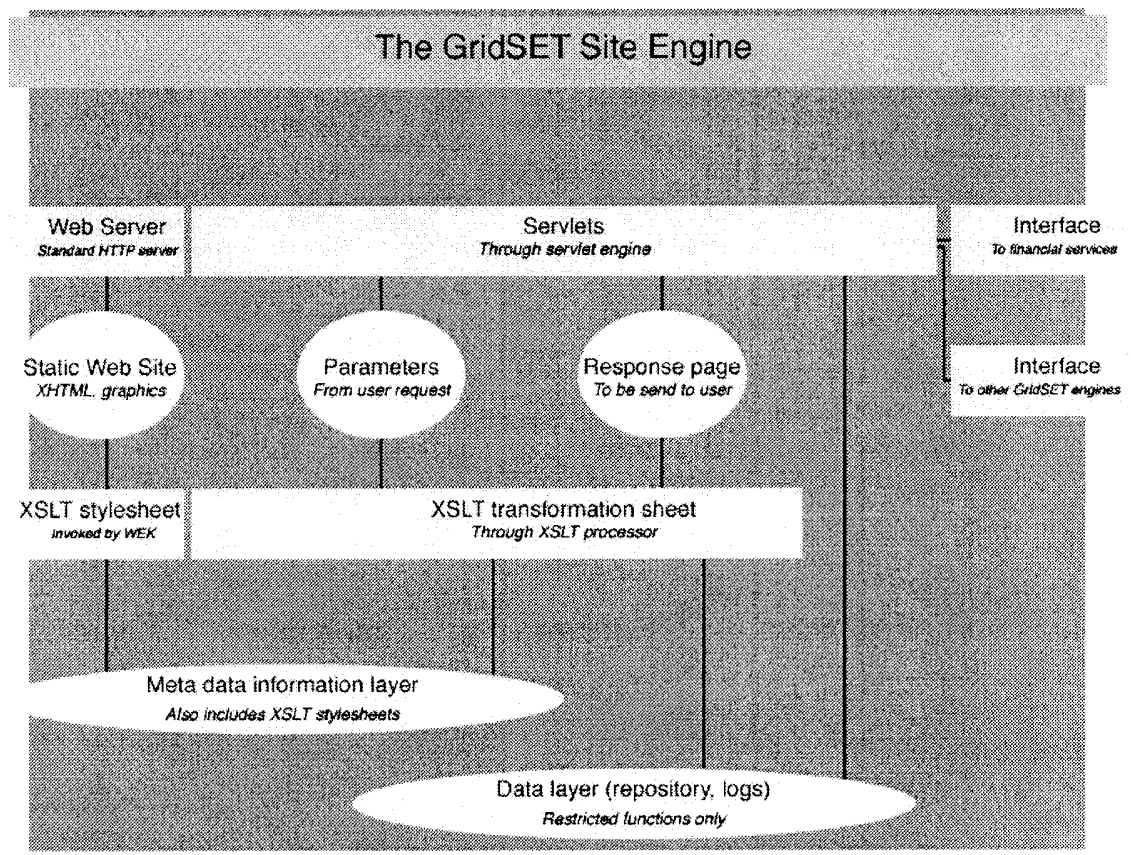

The static web site is created by applying XSLT stylesheets on XML-documents describing the web site content. The lay-out of the dynamic part is also created by XSLT stylesheets.

For the dynamic part, the users sends a request to the Servlet Engine that invokes the appropriate Servlet with the entered user parameters. The Servlet then invokes the XSLT processor to process an XSLT transformation sheet, to process meta-data (read it, update it, etc.) and create a response page for the user. The Servlet Engine then sends this page to the user. This scheme works for instance for a search machine, to get sorted lists of repository items and to update information on organisations and users.

For adding items to the repository, the Servlet and XSLT sheets designed for this purpose have to work closely together. The Servlet.gets the upload request from the user, runs an XSLT stylesheet to check the user's credential and determine where to store the object. Then it stores the object in the repository, runs an XSLT sheet to update the meta-data layer and then sends a response back. 
To delete an item from a repository, save-guards ware build in, to prevent illegal deleting.

Presenting a catalogue in different views on the web is rather straight forward. The advantage of using MPEG-7 as our basic format is that we can use XPath together with the XSLT string functions to create a fine grained search engine. This search engine is then used when creating views for the user.

\section{The design of the editor}

Is getting information out of the system through a web interface not a challenge, getting information in, is. We set out with a client in mind that only had an HTML browser. Reason is that this is the only thing that is guaranteed to work anywhere, anytime. With JavaScript, a much more sophisticated interface can be built, but, unfortunately, the JavaScript implementation by the different browser vendors are too different. Using a Java Applet, is a little better, as long as one restricts to using Java 1.1. But then the interface is not much better than with pure HTML with CSS. XForms, will certainly help, but as the standard is not ready, no browser has implemented it to date.

Hence, we use for an editor, that is completely presented in HTML, with additional JavaScript in to ease editing, not as an essential part. The editor definitions and configurations are all stored as pure XML, i.e. when for instance X-Forms catch on, only a small part of the presentation layer will have to be rewritten.

Within GridSET, a lot of customised editor screens are needed. For instance for entering a user, specifying object meta data for different types of objects, specifying allowable usage, etc. The editor screens must also be customisable by user profiles.

The editors are created dynamically, bootstrapped by an initial specification process:

The MPEG-7 schema fragment describing the information that must be editable is put in an XML-configuration file.

The names (labels) to be used for each MPEG-7 item are specified in another part of the XML-configuration file. This naming scheme can be used to create application and user language (English, Dutch, French, etc.) dependent names.

An XSLT stylesheet automatically creates an initial editor for the schema fragment. In most cases, however, there are parts of the schema that although must be specified, should not be editable for the user. With additional special XSLT-templates in the XSLT-stylesheet these are excluded from the user view (but kept as hidden properties with a specified value.

The layout of the editor screen (width of fields, font-type, colours, etc.) is specified in an XSL-FO (Extensible Stylesheet Language - Formatting Objects) file. This file is used by the XSLT stylesheet producing the user view.

When the user creates or edits information, the data that are send back to the user are also handled by an XSLT-stylesheet using the same MPEG-7 schema fragment as basis. 
Designing editors with XSLT is extremely fast and can make use of the extensibility build in XML. There is one major drawback, however. XSLT can only handle correct conforming XML documents. Users can easily type things that are not conforming XML. Hence, care has been take to exclude that possibility as much as possible, helping the user to write correct XML. MPEG-7 helps in this way because it is a data oriented XML language rather than a document oriented XML language. This means that an editor can consists of fields to be filled in, rather than that is has to be a WYSIWYG document editor.

\section{Conclusion}

With the upcoming $3 \mathrm{G}$ technologies, that adds to the already available user interfaces, we must try to separate content from presentation. In this paper we described an full XML-based approached that can be used for future generation information systems. A market place implementation for medical multimedia objects, called GridSET has been developed. We have also showed that the MPEG-7 standard can be used as a foundation for the XML descriptions.

\section{References}

[1] Genias Virtual Media Publisher, http://www.genias.nl/vmp, last accessed January 2002

[2] The WebSET Consortium project website, http://www.vmwc.org/projects/webset, last accessed January 2002.

[3] Kaplan, S and Sawhney, M. E-Hubs, "The new B2B marketplaces", Harvard Business Review, May-June 200, 97-103.

[4] Long Term Technology

Review of the Science \& Engineering Base, Report to the UK Research Councils, April

[5] MPEG-7, Technology - Multimedia Content Description Interface - Part 5: Multimedia Description Schemes, ISO/IEC 15938, ISO Organisation, Geneva, 2000.

[6] A. Tokmakoff (ed.), State-of-the-Art in Electronic Accouning, Billing and Payment, Enschede, Telematica Instituut http://www.telin.nl, April 18, 2000. 